\title{
EUV Constraints on Models of Low Mass X-Ray Binaries
}

\section{J. CHRISTIAN, ${ }^{1}$ J. E. EDELSTEIN, ${ }^{2}$ M. MATHIOUDAKIS,${ }^{1}$ K. McDONALD, ${ }^{1}$ AND M. M. SIRK ${ }^{1}$}

\author{
${ }^{1}$ Center for EUV Astrophysics, 2150 Kittredge St. Berkeley, CA, 94720-5030, USA \\ ${ }^{2}$ Department of Astronomy, Space Sciences Lab., University of California Berkeley, 94720, USA
}

We present EUVE survey results for moderate column directions containing known low-mass $X$-ray binaries (LMXB). We derive Lexan band $(100 \AA)$ count rates and upper limits for nearly 40 LMXB chosen generally with $E_{B-V} \leq 0.3$. Detections include Sco X-1, Her X-1, and the GRO transient CJ0422+32. Super soft sources in the LMC yield $3 \sigma$ upper limits of $\leq 10$ counts ks-1. The extrapolation of two component spectral models (such as blackbody plus thermal bremsstrahlung), are in agreement with the survey upper limits. Contemporary LMXB spectral models, which involve Comptonization in an inner disk corona, predict a large flux of EUV photons. If the above model is correct in the EUV, such a component could be detected in source with low column densities. We argue that additional intrasystem column hampers its detection.

\section{Introduction}

Low Mass X-ray Binaries (LMXB) with their near Eddington luminosities, X-ray bursts, and dipping behavior provide the opportunity to study accretion onto neutron stars. They are best classified on the basis of their color-color diagrams, which show a Z pattern for the persistently bright sources and less defined patterns (Atoll) for the burst sources (Hasinger \& van der Klis 1989; van der Klis 1989). This spectral behavior is correlated with the short time-scale variability and often show broad features or quasiperiodic oscillations (QPO) in their power spectra. Constructing a physical model of LMXB from the spectral data has been made difficult, due to the limited sensitivity and band-passes of X-ray instrumentation. Models involving a boundary layer plus an accretion disk (Mitsuda 1984) are indistinguishable from Comptonization models (White, Stella, \& Parmar 1989) for many X-ray instruments. Contemporary models have sought to build a self-consistent model to explain both the spectral and QPO behavior. Such models often require a large input of soft $(\leq 1 \mathrm{keV}$ ) photons (Lamb 1989; Schulz \& Wijers 1993).

\section{Observations and Analysis}

EUVE conducted an all-sky survey between July 1992 and January 1993 with three scanning telescopes. Scanners A and B each had two Lexan/boron ("Lexan") quadrants with a bandpass covering $58-174 \AA$ and two $\mathrm{Al} / \mathrm{Ti} / \mathrm{C}$ filters with a bandpass covering 156-234 $\AA$. The bandpasses of scanner C covered $345-605 \AA$ for the $\mathrm{Ti} / \mathrm{Sb} / \mathrm{Al}$ filter, and 500-740 $\AA$ for the $\mathrm{Sn} / \mathrm{SiO}$ filter (Bowyer et al. 1996).

\subsection{Skymaps and Pigeonholes}

We calculated count rates from the EUVE all-sky survey for J2000 positions taken from the White, Giommi, \& Angelini catalogue for known LMXB generally with $E_{B-V}$ less 
than 0.3 (van Paradijs 1993). However, a few globular cluster sources and blackhole candidates with higher $E_{B-V}$ were included. This software produces skymaps, which are a binned distribution of EUV photons for a chosen circular region of the sky. A maximum likelihood technique is used to test for variations above the expected background (Lewis 1993; Bowyer et al. 1996). To verify count rates greater than $3 \sigma$ in the Lexan band skymaps (since we do not expect any real flux above $100 \AA$ for most LMXB) we constructed "pigeonholes" (lists of photon events), with $24^{\prime}$ radius centered on the position of the source. Results from the all-sky survey are shown in Table 1. The likelihood significance quoted in the table is related to the square of the Gaussian significance, $\sigma$. Sources with a downward arrow in Table 1 denote $3 \sigma$ upper limits.

\section{Results}

Sco X-1 and Her X-1, which did appear in the first EUVE All Sky survey (Bowyer et al. 1994) are also reported here showing large Lexan and $\mathrm{Al} / \mathrm{C}$ count count rates. The high column density of Sco X-1 cuts off photons with wavelengths longer than $\sim 64$ $\AA$, therefore the $\mathrm{Al} / \mathrm{Ti} / \mathrm{C}$ result is an X-ray leak. The Lexan count rate for Sco X-1 is some fraction of $\mathrm{X}$-ray leak, since the bandpass does extend down to $30 \AA$ (discussed below). The Lexan count rate for Her X-1 is not suspect, because of its low interstellar column. Her X-1 (although an accreting X-ray pulsar) has been the only X-ray binary EUVE has been able to study in any detail (Vrtilek et al. 1994). The hard transient, GRO CJ0422+32 (Nova Persei 1992) is detected in Lexan at a $4.5 \sigma$ level. Since the first all-sky source catalog use a $6 \sigma$ detection threshold, CJ0422+32 was not included in that catalog. Super-soft source in the LMC (Greiner, Hasinger, \& Kahabka 1991), such as Cal 83, Cal 87, RX J0439.8-6809, and RX J0527.8-695 have upper limits of $\leq 15$ counts per ks.

We have predicted the Scanner Lexan count rates based on $\mathrm{X}$-ray model fits from simultaneous Einstein solid state spectrometer (SSS; $0.5-4.5 \mathrm{keV}$ ) and monitor proportional counter (MPC; $1.2-20.0 \mathrm{keV}$ ) data (Christian 1993). Best fitting spectral models from the Einstein LMXB survey included a form of unsaturated Comptonization (USC; $\mathrm{A} E^{-\mathrm{\Gamma}} \exp (-\mathrm{E} / \mathrm{kT}$ ), where $\Gamma$ is the spectral index), and a blackbody plus thermal bremsstrahlung $(\mathrm{BB}+\mathrm{TB})$. We folded these models through the Lexan effective area. The majority of predictions are an order of magnitude lower than the survey upper limits. Less than $10 \%$ of the observed Lexan flux is expected for the X-ray model of Sco X-1. A large fraction of the counts are from wavelengths shorter than $30 \AA$. The unfolded SSS+MPC model extrapolated to the Lexan bandpass is shown in Figure 1.

\section{Discussion}

Many of the present models of LMXB are based on a possibly non-unique model fits of the X-ray spectra (e.g., Vacca et al. 1987) We have learned models of LMXB must be able to account for QPO, spectral-temporal correlations, and the observed spectra. Contemporary Comptonization models of LMXB have attempted this (Lamb 1989; Ponman, Foster, \& Ross 1990; Schulz \& Wijers 1993). Such models consider a cocoon of Comptonizing material surrounding the neutron star and assume an input spectrum, such as a cutoff power-law or blackbody. The emerging spectrum is a function of the scattering optical depth, which is very sensitive to the mass accretion rate. Such models can produce the observed spectra with a similar number of free parameters as two component models (Ponman, Foster, \& Ross 1990). Phenomenological models of the LMXB spectra 
TABLE 1. EUVE Survey Observations of Low Mass X-ray Binaries.

\begin{tabular}{|c|c|c|c|c|c|c|c|c|}
\hline \multirow[t]{2}{*}{ Source } & \multirow{2}{*}{$\begin{array}{c}\text { Name } \\
(2)\end{array}$} & \multirow{2}{*}{$\begin{array}{c}E_{B-V} \\
(3)\end{array}$} & \multicolumn{3}{|c|}{ Lexan } & \multicolumn{3}{|c|}{$\mathrm{Al} / \mathrm{Ti} / \mathrm{C}$} \\
\hline & & & $\begin{array}{c}S \\
(4)\end{array}$ & Time & $\begin{array}{c}\text { counts ks }{ }^{-1} \\
(6)\end{array}$ & $\underset{(7)}{S}$ & $\underset{(8)}{\text { Time }}$ & $\begin{array}{c}\text { counts ks } \\
\text { (9) }\end{array}$ \\
\hline $0042+327$ & $4 \mathrm{U} 0042+327$ & 0.2 & 0.9 & 1225 & $22 \downarrow$ & 0.4 & 1217 & $28 \downarrow$ \\
\hline $0422+32$ & CJ0422+32 & 0.4 & 22.3 & 702 & $51 \pm 15$ & 7.3 & 694 & $87 \downarrow$ \\
\hline $0439.8-65$ & RXJ0439.8-6 & 0.1 & 6.9 & 8376 & $14 \downarrow$ & 0.4 & 8041 & $17 \downarrow$ \\
\hline $0512-401$ & NGC1851 & 0.1 & 0.0 & 1349 & $15 \downarrow$ & 6.5 & 1343 & $53 \downarrow$ \\
\hline $0521-720$ & LMC X-2 & 0.1 & 1.30 & 9218 & $10 \downarrow$ & 1.2 & 8714 & $16 \downarrow$ \\
\hline $0527.8-69$ & RXJ0527.8-61 & 0.1 & 0.0 & 14201 & $3 \downarrow$ & 2.5 & 13633 & $16 \downarrow$ \\
\hline 0543-682 & CAL 83 & 0.1 & 0.9 & 31555 & $7 \downarrow$ & 0.0 & 30498 & $21 \downarrow$ \\
\hline $0547-711$ & CAL 87 & 0.1 & 1.8 & 13366 & $8 \downarrow$ & 3.5 & 13381 & $16 \downarrow$ \\
\hline $0614+091$ & 4U0614+091 & 0.3 & 1.0 & 988 & $31 \downarrow$ & 3.4 & 942 & $83 \downarrow$ \\
\hline $0620-003$ & V616 Mon & 0.4 & 5.6 & 1188 & $42 \downarrow$ & 0.5 & 1144 & $54 \downarrow$ \\
\hline $0748-767$ & EXO0748-676 & 0.42 & 6.7 & 6495 & $15 \downarrow$ & 6.0 & 6284 & $26 \downarrow$ \\
\hline $0918-549$ & 4U0918-549 & 0.3 & 2.0 & 2386 & $19 \downarrow$ & 4.7 & 2308 & $47 \downarrow$ \\
\hline $0921-63$ & A0921-630 & 0.2 & 1.1 & 3128 & $15 \downarrow$ & 0.4 & 2951 & $25 \downarrow$ \\
\hline $1124-684$ & Nova Mus 91 & 0.25 & 0.3 & 1782 & $22\rfloor$ & 0.9 & 1738 & $54 \downarrow$ \\
\hline $1254-69$ & 4U1254-690 & 0.35 & 6.7 & 508 & $72 \downarrow$ & 0.3 & 491 & $67 \downarrow$ \\
\hline $1617-155$ & Sco X-1 & 0.3 & 792 & 2024 & $284 \pm 16^{a}$ & 273 & 1010 & $248 \pm 23^{a}$ \\
\hline $1627-673$ & $4 U 1627-673$ & 0.1 & 1.3 & 889 & $30 \downarrow$ & 5.1 & 603 & $74 \downarrow$ \\
\hline $1656+354$ & Her X-1 & $\leq 0.05$ & 611 & 1912 & $180 \pm 13$ & 7.0 & 1907 & $42 \downarrow$ \\
\hline $1704+24$ & $\mathrm{~A} 1704+240$ & 0.3 & 3.9 & 1144 & $40 \downarrow$ & 8.2 & 676 & $91 \downarrow$ \\
\hline $1728-169$ & GX $9+9$ & 0.3 & 6.2 & 574 & $75 \downarrow$ & 5.1 & 558 & $104 \downarrow$ \\
\hline $1735-44$ & V926 Sco & 0.15 & 0.2 & 649 & $39 \downarrow$ & 3.8 & 638 & $80 \downarrow$ \\
\hline $1820-303$ & NGC6624 & 0.3 & 1.9 & 1209 & $28 \downarrow$ & 3.4 & 1154 & $75 \downarrow$ \\
\hline $1822-371$ & V691 CrA & 0.15 & 0.0 & 1232 & $15 \downarrow$ & 0.6 & 1160 & $55 \downarrow$ \\
\hline $1832-33$ & NGC6652 & 0.1 & 0.3 & 1032 & $28 \downarrow$ & 0.8 & 970 & $74 \downarrow$ \\
\hline $1908+005$ & Aql X-1 & 0.4 & 7.9 & 1083 & $51 \downarrow$ & 2.1 & 1049 & $71\rfloor$ \\
\hline $1916-053$ & V1405 Aql & 0.2 & 4.5 & 1045 & $45 i$ & 2.0 & 1018 & $66 \downarrow$ \\
\hline $1957+115$ & $4 U 1957+115$ & 0.4 & 6.4 & 773 & $59 \downarrow$ & 0.0 & 712 & $64 \downarrow$ \\
\hline $2127+119$ & M15 & $\leq 0.06$ & 0.9 & 1088 & $30 \downarrow$ & 3.0 & 1023 & $82\rfloor$ \\
\hline $2129+47$ & V1727 Cyg & 0.5 & 1.7 & 1578 & $24 \downarrow$ & 0.2 & 1498 & $31 \downarrow$ \\
\hline $2142+38$ & Cyg X-2 & 0.45 & 6.9 & 1430 & $38 \downarrow$ & 0.3 & 1352 & $44 \downarrow$ \\
\hline
\end{tabular}

Col 1-Source

Col 2-Alternate name

Col 3-E $E_{B-V}$ adapted from van Paradjis 1993.

$\mathrm{Col} 4 \& 7-\mathrm{S}$ is the likelihood significance equal to the square of the Gaussian significance $\sigma$.

Col 5\&8-Exposure Time in seconds.

Col 6-The Lexan/boron count rate per ks. The down arrow $(\downarrow)$ indentifies a $3 \sigma$ upper limit.

Col 9-Same as Column 6 , but for the aluminum/titanium/carbon filter.

${ }^{a} \mathrm{X}$-ray leak.

generally predict 1 to 2 orders of magnitude less EUV luminosity as compared to the $\mathrm{X}$-ray luminosity. This comparison is shown in Table 2.

The Lamb model predicts a large flux of soft photons, possibly from high-harmonic cyclotron emission. If we assume soft photons are produced near the Eddington luminosity for a 1.4 $M_{\odot}$ neutron star and a typical LMXB power-law (with photon index of 2 ), we would expect only $\sim 2$ counts ks ${ }^{-1}$ for a source with a column of $3 \times 10^{21} \mathrm{~cm}^{-2}$ in the Lexan bandpass. For a column which allows photons in the EUVE Lexan bandpass (e.g., $\sim 5 \times 10^{20} \mathrm{~cm}^{-2}$ ) we would expect $\sim 100$ counts $\mathrm{ks}^{-1}$, which would have been 


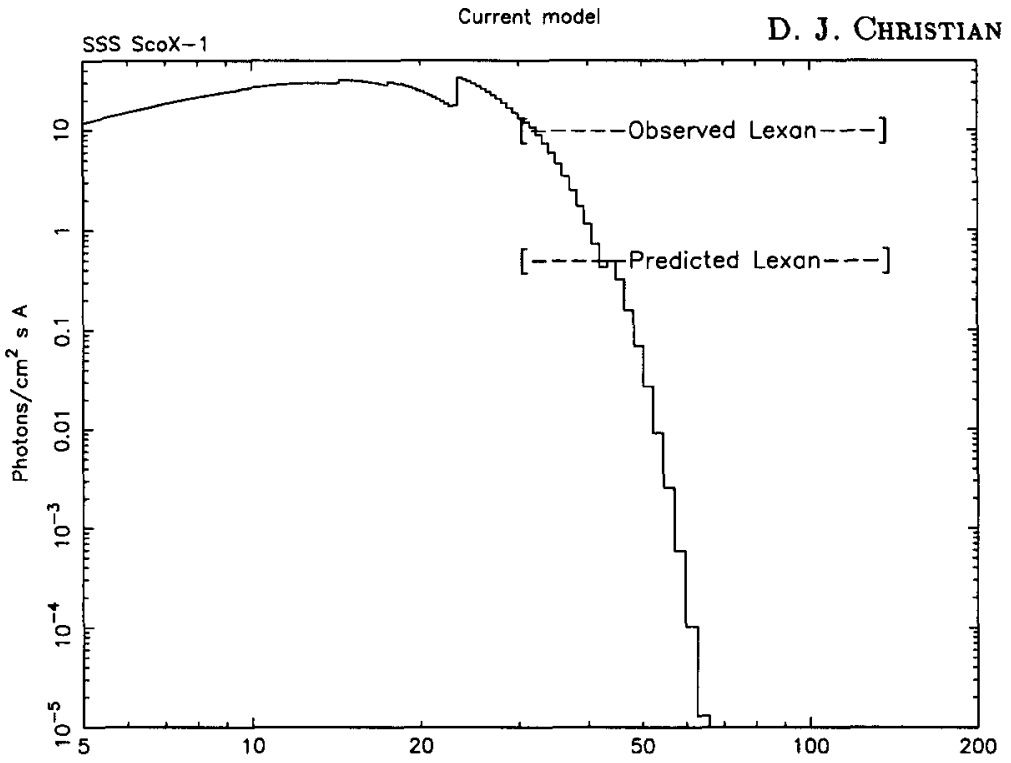

FIGURE 1. The Einstein SSS+MPC model Spetprn of Sco X-1 extraplolated into the Lexan bandpass for comparison. Predicted and Observed Lexan fluxes calculated using the Lexan effective area are indicated.

TABLE 2. Comparison of Lexan and X-ray Luminosities

\begin{tabular}{|c|c|c|c|}
\hline Name & Model & $\begin{array}{l}\text { Luminosity }^{a} \\
\text { Lexan }^{38} \text { ergs s}^{-1} \\
10^{38} \\
\text { (3) }\end{array}$ & $\begin{array}{l}\text { Luminosity } \\
0.5-20.0 \mathrm{kel} \\
10^{38} \mathrm{ergs} \mathrm{s} \\
\text { (4) }\end{array}$ \\
\hline $\begin{array}{l}\text { CJ0422+32 } \\
\text { NGC1851 }\end{array}$ & $\begin{array}{l}\text { CompST } \\
\text { USC }\end{array}$ & $\begin{array}{c}2 \mathrm{e}-4 \\
0.003\end{array}$ & $\begin{array}{c}1.4 \\
0.02\end{array}$ \\
\hline LMC X-2 & USC & 0.1 & 2.0 \\
\hline CAL 83 & $\mathrm{BB}^{c}$ & 0.3 & 0.002 \\
\hline 4U0614+091 & $\mathrm{BB}+\mathrm{USC}$ & $7 e-4$ & 0.02 \\
\hline A0921-630 & USC & $1 e-4$ & 0.01 \\
\hline Sco X-1 & $\mathrm{BB}+\mathrm{TB}$ & 0.03 & 0.7 \\
\hline Her X-1 & $B B+P L^{d}$ & 0.001 & 0.01 \\
\hline NGC6624 & USC & 0.03 & 0.6 \\
\hline X1822-37 & $\mathrm{BB}+\mathrm{TB}$ & $9 \mathrm{e}-6$ & 0.008 \\
\hline M15 & USC & 0.005 & 0.02 \\
\hline $4 U 2129+47$ & $\mathrm{BB}+\mathrm{TB}$ & $2 e-5$ & 0.001 \\
\hline Cyg X-2 & $\mathrm{BB}+\mathrm{TB}$ & 0.07 & 1.4 \\
\hline
\end{tabular}

Col 1-Source

Col 2-The best fitting model from SSS+MPC spectral fits 0.5-20.0 keV (Christian 1993) unless otherwise noted.

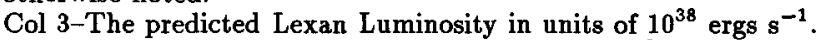

Col 4-The 0.5-20.0 keV Luminosity in units of $10^{38} \mathrm{ergs} \mathrm{s}^{-1}$.

a Luminosites based on distances as compiled in Christian \& Swank 1995.

berived from Griener, Hasinger, \& Thomas 1994

${ }^{c}$ Sunyaev and Titarchuk (1980) form of Comptonization from Pietsch et al. 1993.

${ }^{d}$ From Vrtilek et al. 1994 
detectable in the all-sky survey. Sources like X2127+119 in M15 and X1822-37 fulfill the column density requirement, but are known to have large intrasystem absorption that would absorb most EUV photons. However, the column for X2127+119 does vary with orbital phase (Hertz \& Grindlay 1983), and it could be detectable in the EUV when at the low column phase $(0.0)$. Callanan (this proceedings) report a possible detection of M15 from a pointed $E U V E$ deep survey observation.

This research was supported under NASA contract NAS5-30180.

\section{REFERENCES}

BOWYER, S. ET AL. 1994, ApJS, 93, 569

BOWYER, S. ET AL. 1996, ApJS, in press

Christian, D. J. \& SwANK, J. H. 1995, ApJ, submitted

Christian, D. J. 1993, Ph.D. Thesis, U. of Maryland

Greiner, J., Hasinger, G., \& Thomas, H. C. 1994, A\&A, 281, L61

Hertz, P. \& Grindlay, J. E. 1983, ApJ, 275, 105

HASINGer, G., \& VAN DER Klis, M. 1989, A\&A, 255, 79

LAMB, F. K. 1989, Proc. 23rd ESLAB Symp. 1, 215

Lewis, J. W. 1993, JBIS, 46, 346

MrTsudA, K. 1984, Ph.D. Thesis, U. of Tokyo

Morrison, R., \& McCammon, D. 1983, ApJ, 270, 119

Ponman, T. J., Foster, A. J., \& Ross, R. R. 1990, MNRAS, 246, 287

Schulz, N. S. \& WiJErs, R. A. M. J. 1993, A\&A, 273, 123

Sunyaev, R. A., \& TitarchuK, L. G. 1980, Sov. Astr. L., 12, 117

Vacca, W. D., Sztanjo, M, Lewin, W. H. G., Trumper, J., van Paradius, J., \& Smith, A. 1987, A\&A, 172, 143

van ParadiJs, J, et al. 1990, PASJ, 42, 633

van Paradius, J. 1993, X-ray Binaries, ed. W. H. G. Lewin \& E. P. J. van den Heuvel, Cambridge Univ. Press

VAN DER KLIS, M. 1989, ARA\&A, 27, 517

VRTLEK, S. D. ET AL. 1991, ApJS, 76, 1127

VRTILEK, S. D., ET AL. 1994, ApJL, 436, L9

White, N. E., Peacock, A., \& Taylor, B. G. 1985, ApJ, 296, 475

White, N. E., Stella, L., \& Parmar, A. 1988, ApJ, 324, 363 